\title{
Effectiveness of Carboxymethyl Cellulose for the Removal of Methylene Blue from Aqueous Solution
}

\author{
Hosne Ara Begum* and Mohammad Khalid Bin Mahbub \\ Department of Chemistry, Dhaka University, Dhaka-1000, Bangladesh
}

(Received : 29 August 2012; Accepted : 6 October 2012)

\begin{abstract}
The adsorptive properties of carboxymethyl cellulose (CMC) prepared from sugarcane bagasse (SB) for the removal of methylene blue (MB) from aqueous solution was investigated. Two batches of CMC, CTSB-CMC-B1 and CTSB-CMC-B2, were prepared from chlorite treated sugarcane bagasse (CTSB). The prepared CMCs were characterized by FT-IR spectral analysis. Degree of substitution (DS) value of prepared CMCs was estimated. Batch adsorption experiments show that the adsorption of MB on CMCs reaches equilibrium within 30 minutes. The MB adsorption capacity of CTSB-CMC-B1 and CTSB-CMC-B2 were found to be 652.0 and $369.0 \mathrm{mg} \mathrm{g}^{-1}$, respectively. CMC with the higher DS value (CTSBCMC-B1) shows higher adsorption capacity than the CMC having lower DS value (CTSB-CMC-B2). The uptake of MB was minimum at $\mathrm{pH} 2$ and gradually increases with the increase of $\mathrm{pH}$. From the desorption studies, it was found that large amount of $\mathrm{MB}$ was released in strong acidic ( $\mathrm{pH}$ 3.0) conditions.
\end{abstract}

Key words: CMC, DS value, batch adsorption experiments, adsorption capacity.

\section{Introduction}

Waterways have been used as dumping grounds for industrial effluent since the time of the industrial revolution. However today, because of the sheer volume of waste dumped into our waterways, the natural regeneration capacities of these waterways are being overwhelmed. This poses an environmental threat which directly affects humankind's ability to build a sustainable habitat. Therefore, wastewater decolourisation processes have been the subject of recent research. Dye removal is a complex and expensive process. Methods such as chemical and electrochemical oxidation, membrane filtration, coagulation-flocculation, adsorption and ion exchange are recommended ${ }^{1}$. Amongst the several dye removal techniques, adsorption is considered to give the best results as it can be used to remove different types of coloring materials and organic pollutants. Nowadays, a good number of low cost, commercially available adsorbents have been used for the removal of dyes from coloured wastewater ${ }^{2}$. However, the adsorption capacities of these adsorbents are not satisfactory. The new effective adsorbents which are low-cost and biodegradable are highly demanded.

Sugarcane bagasse is the fibrous residual material of the sugarcane stems left after the crushing and extraction process from sugar mills, which normally accounts for $20-24 \%$ of the cane $^{3}$. In Bangladesh, about 21 million metric tons of bagasse is produced per year as by-product of sugar mills ${ }^{4}$. Sugar cane bagasse contains about $30-50 \%$ of cellulose and $20-24 \%$ of lignin. Some studies on adsorption of different dyes onto sugarcane bagasse have been reported recently $y^{5-9}$. To improve the adsorptive properties of cellulose, its structure is modified to increase its active sites for adsorption of dyes. Currently the most widely adopted methods for modification of cellulose based materials include etherification, esterification, grafting, amination, etc. Carboxymethyl cellulose (CMC) is a cellulose derivative with carboxymethyl groups $\left(-\mathrm{CH}_{2}-\mathrm{COOH}\right)$ bound to some of the hydroxyl groups of the glucopyranose monomers that make up the cellulose backbone (Fig. 1). A number of reports have been published on the adsorption of dye from aqueous solution on $\mathrm{CMC}^{10-12}$.

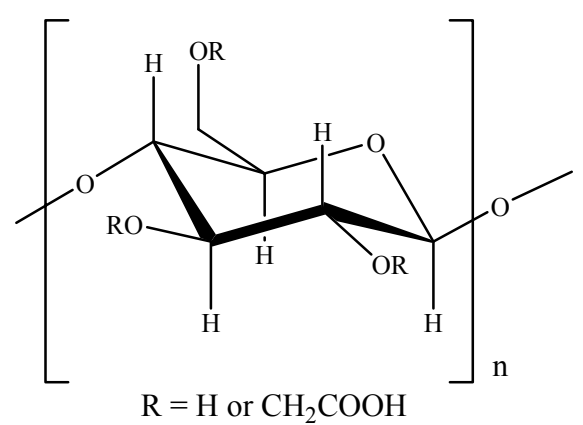

Fig. 1. Structure of carboxylmethyl cellulose<smiles>CN(C)c1ccc2c(c1)S(Cl)=c1cc(N(C)C)ccc1=N2</smiles>

Fig. 2. Structure of methylene blue (MB)

Keeping all these in mind, the aim the present work was to study the effectiveness of partially carboxylmethylated cellulose as a biosorbent for the removal of meythlene blue (MB) from aqueous solution. Meythlene blue, a cationic dye, was selected as a model pollutant (Fig. 2). Sorption experiments included effect of $\mathrm{pH}$, sorption equilibrium, effect of initial concentration and desorption study of MB from MBadsorbed carboxymethyl cellulose under different $\mathrm{pH}$ conditions.

\section{Experimental}

All chemicals and solvents used in the present work were analytical grades (Merck and $\mathrm{BDH}$ ). All solvents were distilled before use. Different solutions and reagents were

*Author for Correspondence, e-mail: hosnearabegum@yahoo.com 
prepared using analytical procedure. Sugarcane bagasse was procured from a sugarcane mill of Bangladesh. The sample was dried in the oven at $45^{\circ} \mathrm{C}$ for 72 hours. After drying, the dried materials were grinded with grinder mill and sieved to a size between 0.500 to $0.212 \mathrm{~mm}$.

\section{Pretreatment of sugarcane bagasse with sodium chlorite}

Raw sugarcane bagasse powder (SB) was treated with acidified sodium chlorite to remove $\operatorname{lignin}^{13}$. The resultant delignified powder obtained was designated as sodium chlorite treated sugar cane bagasse (CTSB).

\section{Preparation of carboxymethyl cellulose (CMC)}

Sodium chlorite treated sugarcane bagasse (CTSB) was partially chemically modified into a carboxymethylated derivative of cellulose (CTSB-CMC) according to the method described elsewhere ${ }^{14}$. CTSB was steeped in aqueous alcoholic sodium hydroxide solution $(18 \%$, water : alcohol $=$ $1: 4)$ and the reaction mixture was continuously stirred for 2 hours at $30^{\circ} \mathrm{C}$. The cellulose alkali mixture was placed in a thermostatic water bath and the temperature was maintained at $58^{\circ} \mathrm{C}$. Monochloroacetic acid solution ( $80 \%$ in ethanol) was added drop wise to the reaction mixture with occasional stirring. The reaction was then allowed to proceed for the desired length of time. Two batches of CTSB-CMC were prepared and they were labeled as CTSB-CMC-B1 and CTSBCMC-B2. The reaction time was 3.5 and 2.0 hours for CTSBCMC-B1 and CTSB-CMC-B2, respectively. The yield of CTSB-CMC-B1 and CTSB-CMC-B2 was found to be 50 and $60 \%$, respectively.

\section{FT-IR spectral analysis}

The FT-IR spectra of the prepared CMCs were recorded using a FT-IR spectrometer (Shimadzu, FT-IR 8400S, Japan) using $\mathrm{KBr}$ pallets in the range of $400-4000 \mathrm{~cm}^{-1}$ with $2 \mathrm{~cm}^{-1}$ resolution.

\section{Determination of degree of substitution (DS) value of CMC}

Degree of substitution (DS) of the prepared CMC samples was determined by $\mathrm{pH}$ titration ${ }^{15}$. $\mathrm{pH}$ vs. volume of sodium hydroxide titration curves (smoothing $1^{\text {st }}$ derivative $\& 2^{\text {nd }}$ derivative) were obtained from experimental data and analyzed by using software CurTipot ( $\mathrm{pH}$ and acid base titration curve: Analysis and simulation) version 3.3.1 (2008) for MS Excel ${ }^{16}$.

\section{Sorption experiments for removal of $M B$}

The stock solution of MB was prepared by dissolving accurately weighed dye in deionized double distilled water (DDW). The experimental solutions were obtained by diluting the stock solution $\left(1000 \mathrm{mg} \mathrm{L}^{-1}\right)$ in appropriate proportions to the required concentrations $\left(2-700 \mathrm{mg} \mathrm{L}^{-1}\right)$. A calibration curve was prepared by recording the absorbance values for MB solutions of known concentrations $\left(2-10 \mathrm{mg} \mathrm{L}^{-1}\right)$ at $\lambda_{\max }$ $664 \mathrm{~nm}$, using a UV-visible spectrophotometer (Shimadzu $\mathrm{UV}-1650$, Japan) at $30^{\circ} \mathrm{C}$.
To determine the adsorptive properties of the prepared adsorbents, CTSB-CMC-B1 and CTSB-CMC-B2, batch sorption experiments were conducted. $0.03 \mathrm{~g}$ of adsorbent and $40 \mathrm{~mL}$ of MB solution of definite concentration were added in each of the experimental bottles. The bottles were shaken in a thermostatic mechanical shaker at $30^{\circ} \mathrm{C}$ with constant speed (110 rpm). For blank, $0.03 \mathrm{~g}$ of adsorbent and $40 \mathrm{~mL}$ of deionised double distilled water (DDW) were added in a bottle and was shaken for 5 hours at $30^{\circ} \mathrm{C}$. $\mathrm{pH}$ adjustments were made using $\mathrm{HCl}$ and $\mathrm{NaOH}$ solutions and was measured using a digital $\mathrm{pH}$ meter. After a definite interval of time each bottle was withdrawn from the shaker. The supernatant of the bottle was transferred and was centrifuged repeatedly until a clear liquid was obtained. Absorption spectrum of the clear solution was then recorded at $\lambda_{\max } 664 \mathrm{~nm}$, using the UV-visible spectrophotometer indicated above. Finally the amount of MB adsorbed for each experiment, $q\left(\mathrm{mg} \mathrm{g}^{-1}\right)$, was calculated according to the following equation:

$$
q=\frac{\left(C_{0}-C_{e}\right) V}{m}
$$

Where, $C_{0}$ and $C_{e}\left(\mathrm{mg} \mathrm{L}^{-1}\right)$ are the initial and final concentrations of solute in solution, respectively; $V(\mathrm{~L})$ is the volume of solution; and $m(\mathrm{~g})$ is the mass of the adsorbent.

\section{Sorption of $\mathrm{MB}$ at different initial $\mathrm{pH}$ of solution}

The effect of initial $\mathrm{pH}$ of $\mathrm{MB}$ solution on the adsorption on CTSB-CMC-B1 and CTSB-CMC-B2 were investigated, for a range of $\mathrm{pH}$ values between 2.0 to 11.0 . The initial concentration of $\mathrm{MB}$ solutions was $100 \mathrm{mg} \mathrm{L}^{-1}$. The adsorbents were weighed and immersed into $40 \mathrm{~mL}$ of $\mathrm{MB}$ solutions with different initial $\mathrm{pH}$. The bottles were continuously shaken at $30^{\circ} \mathrm{C}$ for 6 hours. Final $\mathrm{pH}$ was measured and the optimum $\mathrm{pH}$ (Figs. 3 and 4) was determined. From the amount of $\mathrm{MB}$ adsorbed at each $\mathrm{pH}$, the effect of $\mathrm{pH}$ on adsorption (Figs. 5 and 6) was investigated.

\section{Estimation of equilibrium time}

The time taken for maximum adsorption of $\mathrm{MB}$ on CTSBCMC-B1 and CTSB-CMC-B2 adsorbents were investigated, using 9.6 and $50.0 \mathrm{mg} \mathrm{L}^{-1} \mathrm{MB}$ solutions. Adsorbents were weighed and immersed into bottles of specified concentration and continuously shaken at $30^{\circ} \mathrm{C}$ and $\mathrm{pH}$. After a definite interval of time, ranging from 5 to $360 \mathrm{~min}$, each bottle was withdrawn from the shaker. The initial and final concentrations of the adsorption experiments were determined by the method mentioned above and the extent of adsorption was calculated based on Eq. (1). Results are presented in Figs. 7 and 8.

\section{Effect of initial MB concentration on sorption}

Adsorption experiments were carried out as indicated above using raw sugarcane bagasse (SB), CTSB-CMC-B1 and CTSB-CMC-B2 for different concentrations of MB solutions 
in the range of $10-663 \mathrm{mg} \mathrm{L}^{-1}$ at $\mathrm{pH} 8$ and $30^{\circ} \mathrm{C}$ with 6 hours continuous shaking. Results are presented in Fig. 9.

\section{Desorption study}

After saturated sorption of $\mathrm{MB}$, the MB-adsorbed CTSBCMC-B1 and CTSB-CMC-B2 were collected and washed by deionized double distilled water (DDW). Each of the MBadsorbed $\mathrm{CMC}$ was then dried at $65^{\circ} \mathrm{C}$ in an oven. The effects of different initial $\mathrm{pH}$ on $\mathrm{MB}$ desorption from $\mathrm{CMCs}$ were studied at room temperature. The desorption medium of definite $\mathrm{pH}$ was prepared using $\mathrm{HCl}$ and $\mathrm{NaOH}$ solutions. Each of MB-adsorbed CMC (MB-CMC) was weighed, and then immerged into the desoprtion medium of definite $\mathrm{pH}$, such as $\mathrm{pH}$ 3.0, 6.5 and 10.0 and was continuously stirred for 6 hours. The final MB concentration of each solution was analyzed spectrophometrically to determine the amount of desorption. The results are presented in Table 1.

\section{Results and Discussion}

\section{Characterization of prepared CMC}

The prepared CMCs were characterized by FT-IR analyses. In the FT-IR spectra of CTSB-CMC-B1 and CTSB-CMC-B2, typical peaks for cellulose were observed. The wide peak at around $3000-3700 \mathrm{~cm}^{-1}$ could be due to the stretching vibrations of $-\mathrm{OH}$ groups. Spectra showed $\mathrm{C}-\mathrm{H}$ stretching vibrations in the range of $2850-2950 \mathrm{~cm}^{-1}$. The peak around $1040 \mathrm{~cm}^{-1}$ corresponded to $\mathrm{C}-\mathrm{O}-\mathrm{C}$ bonds. Despites the cellulose backbone, characteristic absorption bands for $\mathrm{CMC}$ were observed in the spectra. The presence of strong absorption bands at around 1600-1640 $\mathrm{cm}^{-1}$ and 1400-1450 $\mathrm{cm}^{-1}$ were observed due to symmetric and asymmetric vibrations of ionized $-\mathrm{COO}^{-}$group. This indicated that carboxyl groups were grafted onto cellulose backbone when carboxymethylation reaction occurred, and $\mathrm{CMC}$ was prepared successfully.

The DS value of CTSB-CMC-B1 and CTSB-CMC-B2 were found to be 0.09 and 0.06 , respectively. These values provide an idea on the extent of carboxymethyl substitution on the surface of the CTSB, and the subsequent adsorption characteristics based on this value. The DS values of CTSBCMC-B1 and CTSB-CMC-B2 also provide us information on the amount $-\mathrm{COOH}$ group present in per gram of absorbent. CMC having low DS value is water insoluble and suitable as adsorbent for dye removal from aqueous solution in a broader $\mathrm{pH}$ range.

\section{Determination of optimum $\mathrm{pH}$}

Prior to the determination of the equilibrium time for the adsorption of MB on CTSB-CMC-B1 and CTSB-CMC-B2, the optimum $\mathrm{pH}$ of the solution used for adsorption experiments was determined. The results of optimum $\mathrm{pH}$ determination are shown in Figs. 3 and 4. To determine the optimum $\mathrm{pH}$ for adsorption of $\mathrm{MB}$ on CTSB-CMC-B1 and CTSB-CMC-B2, $\mathrm{pH}$ of the solution was varied from 2.0 to 11.0 keeping other experimental conditions same. Change of
$\mathrm{pH}$ of the solution before and after the adsorption was found to be negligible at $\mathrm{pH} 8.2$ for CTSB-CMC-B1 and $\mathrm{pH} 7.5$ for CTSB-CMC-B2. For this reason, $\mathrm{pH} 8$ was selected as working $\mathrm{pH}$ for the adsorption experiments.

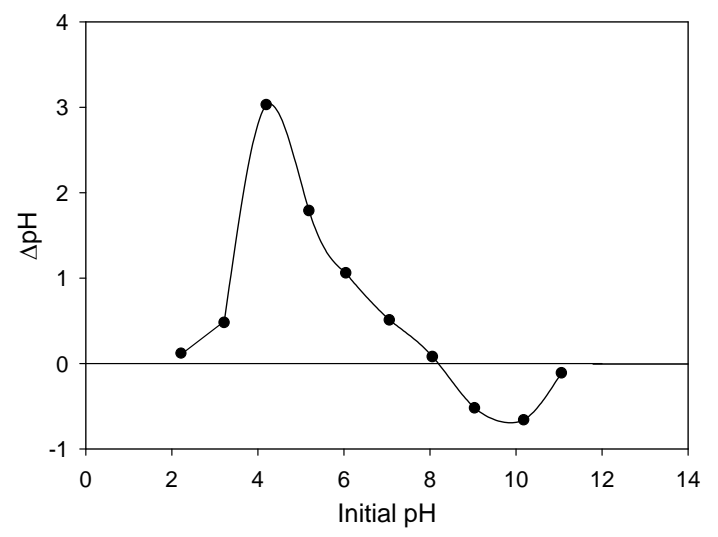

Fig. 3. Determination of optimum $\mathrm{pH}$ of CTSB-CMC-B1 for the adsorption of MB.

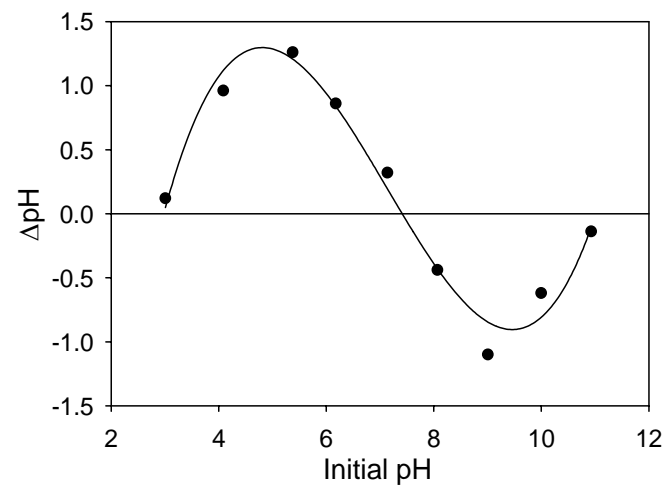

Fig. 4. Determination of optimum $\mathrm{pH}$ of CTSB-CMC-B2 for the adsorption of MB.

\section{Effect of initial $\mathrm{pH}$ on $\mathrm{MB}$ sorption}

The initial $\mathrm{pH}$ of the solution is an important parameter for controlling the adsorption process. The experimental results for the effect of $\mathrm{pH}$ on adsorption of $\mathrm{MB}$ on CTSB-CMC-B1 and CTSB-CMC-B2 are shown in Figs. 5 and 6, respectively. The experimental data shows that the uptake of MB was minimum at around $\mathrm{pH} 2.0$ then gradually increased with the increase of $\mathrm{pH}$. For CTSB-CMC-B1, the uptake gradually reached to maxima near about $\mathrm{pH} 6$, and then remained almost constant over the $\mathrm{pH}$ range of 6.0 to 11.0. Whereas for CTSBCMC-B2, sharp increase was observed at $\mathrm{pH} 4.0$ and adsorption reached to maxima when $\mathrm{pH}$ exceeds 4.0 and then remained almost constant over the $\mathrm{pH}$ range of 5.0 to 11.0. At a lower solution $\mathrm{pH}$, the carboxylic groups of $\mathrm{CMC}$ tended to acquire a net positive charge. As the solution $\mathrm{pH}$ increased, dissociation of carboxylic groups of $\mathrm{CMC}$ occurred and overall charge on CMC surface became negative. This caused an enhancement of attraction between positively charged MB 
and carboxylate anion, resulting in an increase of sorption of $\mathrm{MB}$ onto CMC surface.

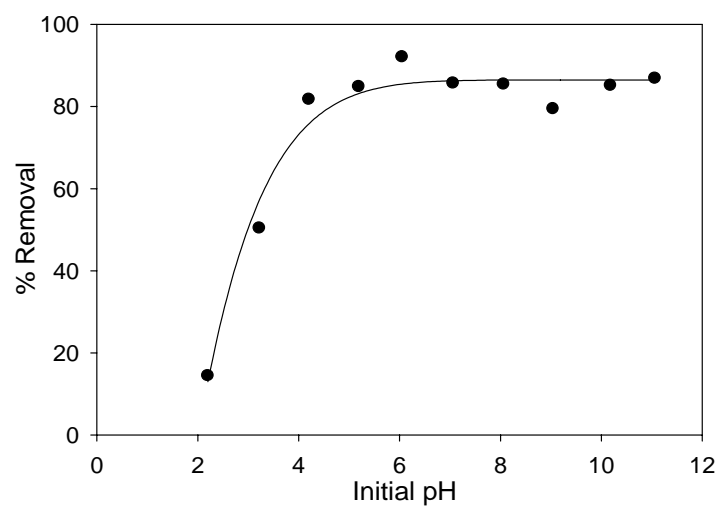

Fig. 5. \% Removal versus Initial $\mathrm{pH}$ of CTSB-CMC-B1.

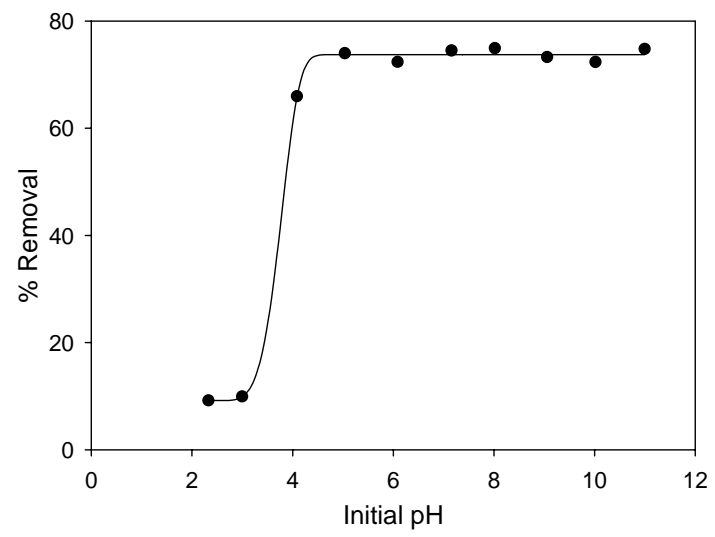

Fig. 6. \% Removal versus Initial pH of CTSB-CMC-B2.

\section{Equilibrium time for sorption}

The sorption-time graph of CTSB-CMC-B1 and CTSB-CMCB2 shown in Figs. 7 and 8, indicates that the adsorption of $\mathrm{MB}$ on $\mathrm{CMC}$ can be considered as a fast adsorption process because more than $70 \%$ of $\mathrm{MB}$ was adsorbed within 10 minutes. Within 30 minutes, adsorption reached to equilibrium. The adsorption process can be divided into three stages: (1) an initial stage with adsorption occurring instantly, (2) subsequently slow adsorption, and (3) a final stage with adsorption reaching equilibrium and remaining constant. The first stage can be attributed to the rapid attachment of MB to the surface of CMC by surface mass transfer. At this stage, more than $70 \%$ of $\mathrm{CMC}$ adsorption was found. The second stage was slower, possibly because many of the available external sites were already occupied and because of the slow diffusion of $\mathrm{MB}$ molecules into the pore spaces of $\mathrm{CMC}$. At final stage, the amount adsorbed did not vary significantly at contact time longer than the equilibrium time. It is evident from Figs. 7 and 8 that the time required to reach an equilibrium concentration $\left(C_{e}\right)$ increases with the increase of the initial $\mathrm{MB}$ concentration $\left(C_{0}\right)$.

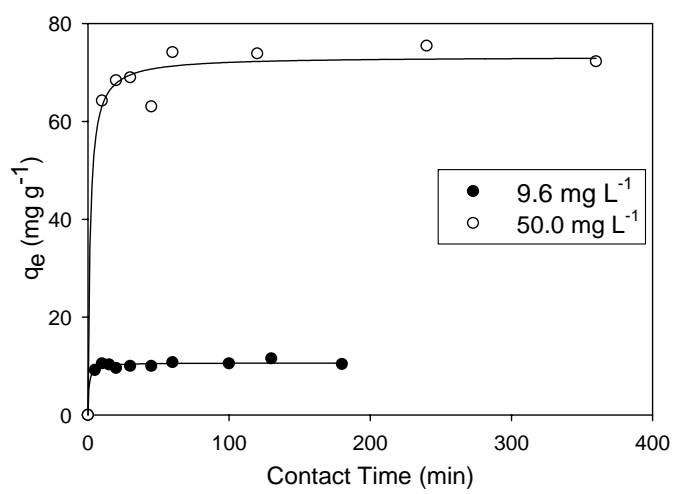

Fig. 7. Comparison of equilibrium time of CTSB-CMC-B1 in different concentrations of $\mathrm{MB}$ solutions at $\mathrm{pH}$ 8.0.

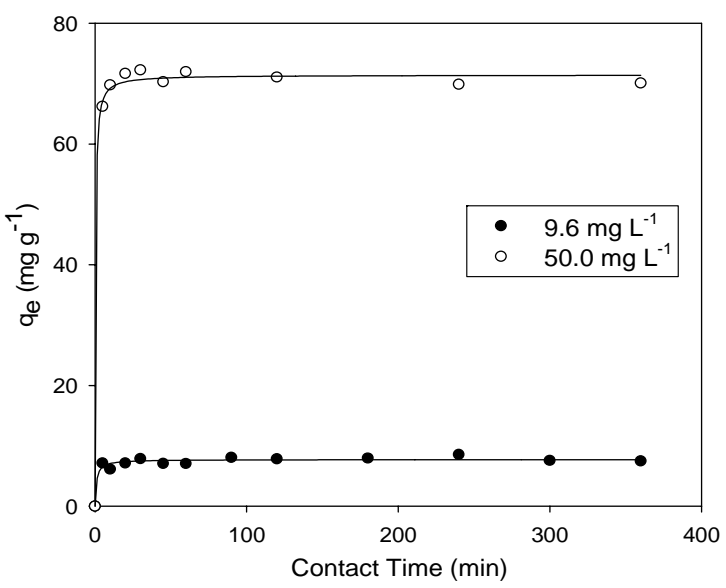

Fig. 8. Comparison of equilibrium time of CTSB-CMC-B2 in different concentrations of $\mathrm{MB}$ solutions at $\mathrm{pH}$ 8.0.

\section{Effect of initial MB concentration}

The influence of initial dye concentration on equilibrium uptake of MB by CTSB-CMC-B1 and CTSB-CMC-B2 is presented in Fig. 9. Results show that increased initial MB concentration led to increased MB adsorption. Apparently, the initial $\mathrm{MB}$ concentration plays an important role in affecting the capacity of CMC sample to adsorb MB. The higher the $\mathrm{MB}$ concentration, the stronger is the driving force of the concentration gradient, and therefore the higher is the adsorption capacity. The MB adsorption capacity of CTSBCMC-B1 and CTSB-CMC-B2 were found to be 652.0 and $369.0 \mathrm{mg} \mathrm{g}^{-1}$, respectively (at initial concentration of $\mathrm{MB}$ solution $=663 \mathrm{mg} \mathrm{L}^{-1}, \mathrm{pH}=8$ and temperature $=30^{\circ} \mathrm{C}$ ). It is observed that the CMC with the higher DS value, i.e. CTSBCMC-B1 with DS $=0.09$, shows higher adsorption capacity than CTSB-CMC-B2 with DS $=0.06$, due to the presence of higher number of $-\mathrm{COOH}$ group, as shown in Fig. 9. The adsorption capacity of MB on CTSB-CMC-B1 and CTSBCMC-B2 was much greater than that of raw sugarcane bagasse (SB) (Fig. 9), which indicates that carboxyl groups on $\mathrm{CMC}$ enhanced the sorption capacity to a great extent. The amount of $-\mathrm{COOH}$ group plays a vital role in adsorption process. A possible mode of $\mathrm{MB}$ binding on $\mathrm{CMC}$ due to 
electrostatic attraction is shown in the Fig. 10. Ion exchanging mechanism of MB sorption on $\mathrm{CMC}$ is presented here.

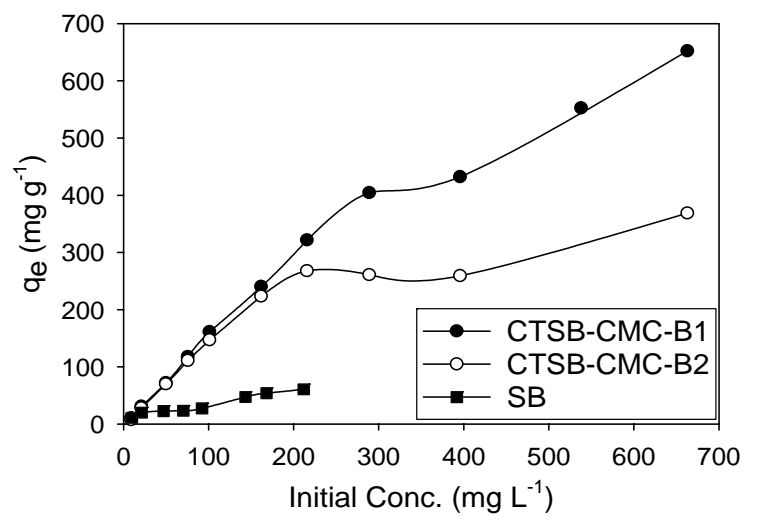

Fig. 9. Amount MB adsorbed on CTSB-CMC-B1, CTSB-CMC-B2 and $\mathrm{SB}$ at different initial concentrations

$$
\left\{-\mathrm{COO}^{-} \mathrm{Na}^{+}+\mathrm{MB}^{+} \longrightarrow\left\{-\mathrm{COO}^{-} \mathrm{MB}^{+}+\mathrm{Na}^{+}\right.\right.
$$

Fig. 10. A possible mode of $\mathrm{MB}$ binding on $\mathrm{CMC} . \mathrm{MB}^{+}$represents the cationic part of MB.

\section{Desorption Study}

It is very important to regenerate the used sorbents in practice applications. However, before this application, the stability of the new sorbents should be checked first. Desorption study of $\mathrm{MB}$ from MB-adsorbed $\mathrm{CMCs}$ (MB-CMC) in desorption medium of $\mathrm{pH} 3.0,6.5$ and 10.0 were carried out at room temperature. If the adsorbed dyes can be desorbed using pure water, the attachment of the dye to the adsorbent is due to weak bonds. If acid or alkaline water desorbs the dye then the adsorption is by ion exchange. If organic acids, like acetic acid can desorb the dye then the dye is held by the adsorbent through chemisorption. The $\mathrm{pH}$ dependence of $\mathrm{MB}$ desorption from MB-CMC is shown in Table 1. It was found that large amount of $\mathrm{MB}$ was released in strong acidic conditions, which was in accordance with previous MB sorption study ${ }^{11}$. It was suggested that MB-CMC was stable enough in the medium having $\mathrm{pH}$ higher than 3.0. Under acidic condition, $\mathrm{CMC}$ with the higher DS value (CTSB-CMC-B1) showed higher desorption ability than that of the low DS value (CTSB-CMCB2).

Table 1. Data for the determination of the amount of MB desorbed for CMC

\begin{tabular}{|c|c|c|c|}
\hline \multirow{2}{*}{$\begin{array}{c}\text { No. of } \\
\text { Obs. }\end{array}$} & \multirow{2}{*}{$\begin{array}{c}\text { pH of } \\
\text { desorption } \\
\text { Medium }\end{array}$} & $\begin{array}{c}|c| \\
\text { CTSB- } \\
\text { CMC-B1 }\end{array}$ & CTSB-CMC-B2 \\
\hline 1 & 3.0 & 72.4 & 54.0 \\
\hline 2 & 6.5 & 4.5 & 8.1 \\
\hline 3 & 10.0 & 3.8 & 4.5 \\
\hline
\end{tabular}

\section{Conclusions}

The experimental results in batch adsorption studies, indicate that carboxymethyl derivatives of cellulose (CMC) prepared from sugarcane bagasse were effective sorbents for removal of $\mathrm{MB}$ from aqueous solutions. The sorption rate of $\mathrm{MB}$ on CMCs was very fast and reaches to sorption equilibrium within 30 minutes. The MB adsorption capacity of CTSBCMC-B1 and CTSB-CMC-B2 were found to be 652.0 and $369.0 \mathrm{mg} \mathrm{g}^{-1}$, respectively. It was observed that CMC with the higher DS value showed higher adsorption capacity of the $\mathrm{MB}$, due to the presence of higher number of $-\mathrm{COOH}$ group. Increased initial $\mathrm{MB}$ concentration led to increased $\mathrm{MB}$ adsorption. The experimental results showed that $\mathrm{MB}$ sorption was $\mathrm{pH}$ dependent. The uptake of $\mathrm{MB}$ was minimum at around $\mathrm{pH} 2.0$ then gradually increased with the increase of $\mathrm{pH}$. It was found that large amount of $\mathrm{MB}$ was released in strong acidic conditions. CMC with the higher DS value showed higher desorption ability under acidic condition. The ability of the CMC adsorbents to undergo desorption had given it the unique ability to be reused, thereby decreasing the cost of industrial effluent treatment. Finally, it may be suggested that CMC with higher DS value removes cationic dye more effectively from the aqueous solution at higher $\mathrm{pH}$.

\section{Reference}

1. Robinson, T., G. McMullan, R. Marchant and P. Nigam, 2001. Remediation of dyes in textiles effluent: a critical review on current treatment technologies with a proposed alternative. Bioresour. Technol., 77, 247-255.

2. Gupta, V. K. and Suhas, 2009. Application of low-cost adsorbents for dye removal - A review. J. Environ. Manage., 90, 2313-2342.

3. Barnes, A. C., 1974. The Sugar Cane. Wiley, New York.

4. Asadullah, M., M. A. Rahman, M. M. Ali, M. S. Rahman, M. A. Motin, M. B. Sultan and M. R. Alam, 2007. Production of biooil from fixed bed pyrolysis of bagasse. Production of bio-oil from fixed bed pyrolysis of bagasse. Fuel, 86, 2514-2520.

5. Raghuvanshi, S. P., R. Singh and C. P. Kaushik, 2004. Kinetic study of methylene blue dye bioadsorption on bagasse. Appl. Ecol. Env. Res., 2, 35-43.

6. Azhar, S. S., A. G. Liew, D. Suhardy, K. F. Hafiz and M. D. I. Hatim, 2005. Dye removal from aqueous solution by using adsorption on treated sugarcane bagasse. Am. J. Appl. Sci., 2, 1499-1503.

7. Abdullah, A. G. L., M. A. M. Salleh, M. K. S. Mazlina, M. J. M. M. Noor, M. R. Osman, R. Wagiran, and S. Sobri, 2005. Azo dye removal by adsorption using waste biomass: sugarcane bagasse. Int. J. Eng. Technol., 2, 8-13.

8. Raymundo, S., R. Zanarotto, M. Belisário, M. D. G. Pereira, J. N. Ribeiro and A. V. F. N. Ribeiro, 2010. Evaluation of sugarcane bagasse as bioadsorbent in the textile wastewater treatment contaminated with carcinogenic Congo Red dye. Braz. Arch. Biol. Technol., 53, 931-938.

9. Elhami, S., H. Faraji and M. Taheri, 2012. Removal of neutral red dye from water samples using adsorption on bagasse and sawdust. J. Chem. Soc. Pak., 34, 269-271. 
10. Borsa, J., F. Kálmán and I. Rusznák, 1989. Equilibrium sorption of crystal violet by carboxymethylated cellulose with low degree of substitution. Colloid Polym. Sci., 267, 915-922.

11. Yan, H., W. Zhang, X. Kan, L. Dong, Z. Jiang, H. Li, H. Yang and R. Cheng, 2011. Sorption of methylene blue by carboxymethyl cellulose and reuse process in a secondary sorption. Colloids Surf., A: Physicochem. Eng. Aspects, 380, 143-151.

12. Liu, Y., W. Wang, Y. Jin and A. Wang, 2011. Adsorption behavior of Methylene Blue from aqueous solution by the hydrogel composites based on attapulgite. Separ. Sci. Technol., 46, $858-868$.
13. Rowell, R. M., 2005. Handbook of Wood Chemistry and Wood Composites. CRC Press, 62.

14. Jahan, I. A., F. Sultana and M. N. Islam, 2007. Studies on indigenous cotton linters for the preparation of carboxymethyl cellulose. Bangladesh J. Sci. Ind. Res., 42, 29-36.

15. Eyler, R. W., E. D. Klug and F. Diephuls, 1947. Determination of degree of substitution of sodium carboxymethyl cellulose. Anal. Chem., 19, 24-27.

16. Gutz, I. G. R., 2008. CurTipot (pH \& acid base titration curve: Analysis \& simulation) version 3.3.1 for MS Excel. 